UCRL-JC-129251

PREPRINT

\title{
Hot Electron Production and Heating by Hot Electrons in Fast Ignitor Research
}

M. H. Key, K. Estabrook, B. Hammel, S. Hatchett, D. Hinkel, J. Kilkenny, J. Koch, W. Kruer, B. Langdon, B. Lasinski, R. Lee, B. MacGowan, J. Moody, M. Moran, A. Offenberger, D. Pennington, M. Perry, T. Phillips, C. Sangster, M. Tabak, G. Tietbohl, M. Tsukamoto, K. Wharton, S. Wilks

This paper was prepared for submittal to the 39th Annual Meeting of the American Physical Society

Division of Plasma Physics

Pittsburgh, PA

November 17-21, 1997

December 1, 1997

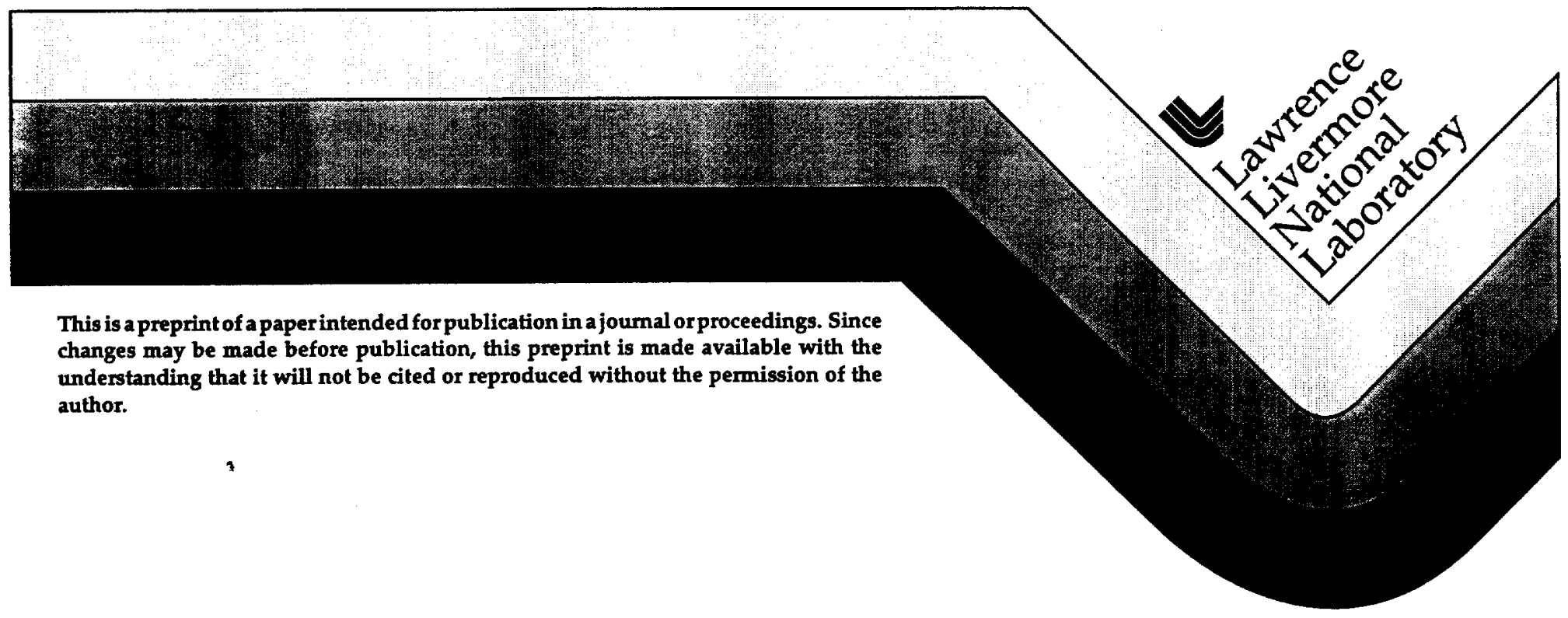




\section{DISCLAIMER}

This document was prepared as an account of work sponsored by an agency of the United States Government. Neither the United States Government nor the University of California nor any of their employees, makes any warranty, express or implied, or assumes any legal liability or responsibility for the accuracy, completeness, or usefulness of any information, apparatus, product, or process

disclosed, or represents that its use would not infringe privately owned rights. Reference herein to any specific commercial product, process, or service by trade name, trademark, manufacturer, or otherwise, does not necessarily constitute or imply its endorsement, recommendation, or favoring by the United States Government or the University of California. The views and opinions of authors expressed herein do not necessarily state or reflect those of the United States Government or the University of California, and shall not be used for advertising or product endorsement purposes. 


\title{
Hot electron production and heating by hot electrons in fast ignitor research
}

M. H. Key K. Estabrook, B. Hammel , S. Hatchett, D. Hinkel, J. Kilkenny , J. Koch, W. Kruer, B. Langdon, B. Lasinski, R. Lee, B. MacGowan, J. Moody, M. Moran, A. Offenberger, D. Pennington, M. Perry, T. Phillips, C. Sangster, M. Tabak, G. Tietbohl, M Tsukamoto, K. Wharton, S. Wilks.

Lawrence Livermore National Laboratory, P.O. Box 808, L-488, Livermore CA 94550

\begin{abstract}
In an experimental study of the physics of fast ignition the characteristics of the hot electron source at laser intensities up to $10^{20} \mathrm{Wcm}^{-2}$ and the heating produced at depth by hot electrons have been measured. Efficient generation of hot electrons but less than the anticipated heating have been observed.
\end{abstract}

The concept of isochoric fast ignition originated by Tabak et al. ' is of importance through its potential to give higher inertially confined fusion (ICF) gain than isobaric central spark ignition used in the more developed indirect and direct drive schemes ${ }^{2}$ and thereby to reduce the driver efficiency required for inertial fusion energy (IFE). The physics is new and challenging involving strongly relativistic laser plasma interactions and transport of energy by $\mathrm{MeV}$ electrons where electrostatic potentials and self generated magnetic fields may strongly modify the transport ${ }^{3}$. Experimental and theoretical studies aimed at assessing the feasibility of fast ignition as a new route to ICF are now being carried out at many laboratories world wide including the Lawrence Livermore National Laboratory (LLNL), where the Nova laser facility has been adapted to generate petawatt pulses using chirped pulse amplification (CPA) ${ }^{4}$.

\section{Experimental Facility}

Two beam lines at Nova have been adapted for CPA operation and for experiments reported here, generated typically $20 \mathrm{~J}$ and $500 \mathrm{~J}$ pulses respectively of duration in the range 0.4 to 20 ps (maximum power up to $1 \mathrm{PW}$ ). Focusing of the two beams respectively was with an off axis $\mathrm{f} / 3$ parabolic mirror of focal length $42 \mathrm{~cm}$ in a focal spot of $15 \mu \mathrm{m}$ diameter 
and an axial $\mathrm{f} / 4$ parabola of focal length $170 \mathrm{~cm}$ in an asymmetrical spot of $40 \mu \mathrm{m} \times 20 \mu \mathrm{m}$

The focal spots had a speckle pattern sub structure with a broad power spectrim of intensity. Work is in progress to correct the wavefront using a deformable mirror. The 20 beam line produced a power weighted average intensity on target in 0.45 ps pulses estimated at $210^{19} \mathrm{Wcm}^{-2}$. The $500 \mathrm{~J}$, beam line produced $10^{20} \mathrm{Wcm}^{-2}$ in $1 \mathrm{PW}, 0.45 \mathrm{ps}$ pulses. A thick glass plate debris shield protected the parabola in the $500 \mathrm{~J}$ beam line for longer pulse operation down to $5 \mathrm{ps}$. Non linear effects precluded the use of the debris shield for 1PW shots and here a plasma mirror was used to reverse the beam direction thus projecting ablated target debris away from the un-protected parabola. The off axis parabola was used with a thin debris shield for all pulse lengths in the $20 \mathrm{~J}$ experiments. Targets in these experiments were exposed to ASE and leakage prepulses before the main pulse. ASE in a typically 3 ns period before the pulse varied in experiments reported here from $210^{-5}$ to $210^{-4}$ of the main pulse energy. The energy of leakage pulses ranged from $10^{-4}$ to $10^{-2}$ of the main pulse, occurring 2 ns or 4 ns before the main pulse but could be made as low as $10^{-6}$ with precise adjustment of Pockels cell gates.

\section{The hot electron source}

\section{$\underline{\mathrm{K} \alpha \text { spectroscopy }}$}

A central theme of the experimental work has been the characterization of the hot electron source produced at a solid target. Electrons directed into the target are required in fast ignition to heat compressed (200 to $600 \mathrm{~g} / \mathrm{cc}$ ) thermonuclear fuel to ignition conditions (15 $\mathrm{keV}$ and density radius product $p r$ of $0.5 \mathrm{gcm}^{-2}$ ). Electrons of about $1 \mathrm{MeV}$. energy have the necessary range.

Extensive experiments were carried out with the $20 \mathrm{~J}, 0.4 \mathrm{ps}$ beam at 25 degree $\mathrm{P}$ polarized incidence on flat layered targets. A Mo fluor layer 50 micron thick was sandwiched 
between a front layer of variable material and thickness and a $1 \mathrm{~mm}$ thick polymer back layer. Electrons from the focal spot on the front layer penetrated into the target and induced $\mathrm{K} \alpha$ fluorescence in the Mo which was recorded via the back of the target by a CCD camera operating as a proportional counter at signal levels giving single photon detection in each pixel . The X-ray spectrum in the vicinity of the $17.5 \mathrm{keV} \mathrm{Mo} \mathrm{K \alpha}$ fluorescence was recorded as a spectrum of counts from the CCD pixels and showed the $\mathrm{K} \alpha$ line with a background continuum. Subtraction of the continuum gave the $K \alpha$ yield. The detector was calibrated absolutely with a $22 \mathrm{keV}, \mathrm{Cd}^{109}$ gamma radiation source.

Data were recorded for $\mathrm{CH}, \mathrm{Al}$ and $\mathrm{Cu}$ front layers of mass per unit area up to $0.5 \mathrm{gcm}^{-2}$, the penetration depth needed for fast ignition. The results ${ }^{5}$ are shown in figures 1 and 2 where the depth at which the fluor layer is located is specified in units of mass per unit area because the range of an electron of a given energy is determined principally by the mass per unit area irrespective of the material. The most obvious features are the decrease in $\mathrm{K} \alpha$ yield with increasing depth in all cases and the decrease at a fixed depth as the material is changed from $\mathrm{Cu}$ to $\mathrm{Al}$ to $\mathrm{CH}$. The electrons are clearly less able to penetrate the $\mathrm{CH}$ target. There is a smaller reduction in penetration changing from $\mathrm{Cu}$ to $\mathrm{Al}$.

To interpret these experiments we used two codes ITS and Lasnex. The first is a 3D Monte Carlo electron photon transport model describing scattering, energy loss and $K \alpha$ and Bremsstrahlung emission by electrons in cold matter with no self consistent potentials or magnetic fields or heating effects. The source of electrons was specified as a relativistic Maxwellian of variable mean energy with either a 90 degree or a 30 degree cone half angle. The second is plasma code Lasnex, which was run in 1D with hydro - effects switched off to describe how hot electrons created with a Maxwellian energy spectrum in a spherical 
region of diameter equal to the focal spot diameter, diffuse and heat up the surrounding initially cold matter. The model computes the changing temperature and resistivity of the plasma, energy loss and heating from electron collisions, electrostatic potential, return current and ohmic heating. The 1D spherical geometry is only a rough approximation to the experiment and the Lasnex results are not therefore suitable for quantitative comparison with experiments. Potentials computed with Lasnex were comparable to the mean energy of the hot electrons only close to the focal spot and at early times. The potential well rapidly increased in radius and decreased in amplitude and was of negligible significance over most of the distance traversed by the electrons in diffusing to the fluor layer. The computed behavior for $\mathrm{Cu}, \mathrm{Al}$ and $\mathrm{CH}$ targets was similar but the modelling of $\mathrm{CH}$ targets was more inaccurate as the high resistivity of cold $\mathrm{CH}$ was seriously underestimated by the plasma model. Ohmic j.E heating within the potential well was predicted to be significant accounting for example for $38 \%$ of the absorbed laser energy in $\mathrm{Cu}$ targets.

Based on the Lasnex modelling, use of the ITS code to model the $\mathrm{K} \alpha$ data for $\mathrm{Cu}$ and $\mathrm{Al}$ targets was an approximation which was expected to be valid over most of the path traversed by the electrons. The code was run for a relativistic Maxwellian electron energy distribution function which was found to give a good fit to PIC code modeling ${ }^{6}$. The ITS model showed that typically $90 \%$ of the excitation of $\mathrm{K} \alpha$ fluorescence was by electron collisions and $10 \%$ by the Bremsstahlung radiation from the hot electrons. Fitting the slope of the fall off in $\mathrm{K} \alpha$ yield with depth gave the mean energy $\langle E\rangle$ or temperature $k T$ of the hot electrons. Fitting the absolute yield gave a conversion efficiency which was a weak function of the angular distribution of the source electrons. For $\mathrm{Cu}$ targets $\langle\mathrm{E}\rangle=0.64 \pm 0.2$ $\mathrm{MeV}$ and a conversion efficiency of $28 \%$ were obtained assuming a 90 degree cone half angle. The corresponding figures for a 30 degree half angle were a lower efficiency of $21 \%$ at the same mean energy. The data for $\mathrm{Al}$ had less scatter and assuming a 90 degree cone 
half angle, were fitted with $\langle E\rangle=330 \pm 120 \mathrm{keV}$ and an efficiency of $31 \%$, the latter only marginally different from the $\mathrm{Cu}$ result within the errors. The estimated conversion efficiency is a lower bound since the K $\alpha$ measurement is not sensitive to the energy loss of the electrons to the induced potential which is recovered as ohmic $j . E$ heating. An estimate of the necessary scale up in conversion efficiency is given by the ratio $(E o+E c) / E c$ for the ohmic ' $\mathrm{o}$ ' and collisionally deposited 'c' energies. For $\mathrm{Cu}$ this factor would give a 1.6 fold efficiency increase if the 1D model were exact. 2D modelling is needed however to give a quantitatively useful scaling factor.

The $\mathrm{CH}$ data are radically different and show a strong reduction of electron penetration.We tentatively attribute this to potentials generated by the electron flow in the high resistivity of cold $\mathrm{CH}$ which is not well modeled in Lasnex.

The discussion so far neglects magnetic fields which may modify the electron transport. For instance we estimate that the hot electron current would be 1000 times the Alfven current if not neutralised by a cold electron return current. A reduction of electron penetration by magnetic fields would lead to a further underestimate of the conversion efficiency and/or mean energy. Our estimates should for this reason also be regarded as a lower bound.

We quote mean energy ( $640 \mathrm{keV}$ for $\mathrm{Cu}$ ) in preference to the temperature of the electrons for the following reasons. The temperature of a fully relativistic Maxwellian has a mean energy of $3 \mathrm{kT}$ whereas for a non relativistic Mawellian the mean energy is $3 / 2 \mathrm{kT}$. Classical Maxwellian or simple exponential forms having the same mean energy (and similar half width) but different temperatures gave essentially the same ITS model predictions for $\mathrm{K} \alpha$ yield. Mean energy therefore seems to be invariant in the fits. PIC modeling ${ }^{6}$ gives a mean energy of $690 \mathrm{keV}$ at $1.210^{19} \mathrm{Wcm}^{-2}$. Scaling in proportion to the relativistic ponderomotive potential (RPP) suggests that $<$ E $>$ given by PIC modelling would be 960 
$\mathrm{keV}$ at $210^{19} \mathrm{Wcm}^{-2}$. This is close to the $930 \mathrm{keV} \mathrm{RPP} \mathrm{at} 210^{19} \mathrm{Wcm}^{-2}$. The $640 \mathrm{keV}$ experimental value is therefore about $70 \%$ of the RPP.

Work elsewhere has determined the temperature of the hot electrons over a range of intensity $x$ (wavelength) ${ }^{2}$ up to mid $10^{18} \mathrm{Wcm}^{-2} \mu \mathrm{m}^{2}$ giving scaling ${ }^{7}$ proportional to (intensity) ${ }^{0.3}$ which extrapolate to $\mathrm{kT}=450 \mathrm{keV}$ at $210^{19} \mathrm{Wcm}^{-2}$. The temperature associated with our fitted $\langle\mathrm{E}\rangle=0.64 \mathrm{MeV}$ is $0.3 \mathrm{keV}$. Our lower bound measurement is therefore lower (70\%) relative to other temperature measurements.

We expect experimental results in general to be significantly influenced by the form of the power spectrum of intensity on the target which for typically many times diffraction limited beams we believe is broad and similar to that in a classical speckle pattern. Relativistic self focusing in preformed plasma ${ }^{8}$ may further broaden the spectrum of intensity. The fraction of the power at the quoted intensity is difficult to determine in practice and differences between experiments may arise from this source.

A new but limited set of data was obtained recently with the high energy beam line. Al targets irradiated by $5 \mathrm{ps}, 300 \mathrm{~J}$ pulses at $10^{19} \mathrm{Wcm}^{-2}$ gave results which although scattered, were roughly fitted by the same mean energy as the results from $20 \mathrm{~J} .0 .4$ ps $\left(210^{19} \mathrm{Wcm}^{-2}\right.$ ) shots, but with a reduced 10 to $15 \%$ conversion efficiency. For longer pulses at lower intensity (20 ps, 200 to $400 \mathrm{~J}$ pulses at $210^{18} \mathrm{Wcm}^{-2}$ ) there was also no apparent change of mean energy but a further reduction of conversion fraction to 5 to $10 \%$. These results show an intensity dependence of the conversion fraction if analyzed assuming a constant mean energy of $330 \mathrm{keV}$ as shown in figure 3 . Our preliminary interpretation of this behavior is that it is attributable to a multi-temperature 'hot tail' form of the electron energy distribution which is also evidenced in hard X-ray Bremsstrahlung spectra discussed below. The low intensity long pulse shots produce an electron distribution in which only the hot tail penetrates to the depth of the $\mathrm{K} \alpha$ measurements 
whereas in the case of the high intensity data the bulk of the electrons can penetrate to that depth. The apparent mean energy for the low intensity shots is therefore associated with the hot tail and we expect that the bulk of the electrons have a lower mean energy.

\section{Bremmstrahlung emission}

Bremsstrahlung emission by the hot electrons gives useful additional information. In the case of thick high $\mathrm{Z}$ targets, Bremsstrahlung from the target itself dominates over that from the target chamber walls. Data of this type were obtained at normal incidence on Au targets with both $5 \mathrm{ps}$ and $0.5 \mathrm{ps}$ pulses from the high energy beam line.

Using the PW, 0.5 ps beam reflected by a plasma mirror, the typical energy on target assuming $70 \%$ plasma mirror reflectivity, was $330 \mathrm{~J}$ and the power weighted mean intensity was up to $10^{20} \mathrm{Wcm}^{-2}$. The forward directed X-ray Bremstrahlung spectrum from $1 \mathrm{~mm}$ thick Au targets was measured at 45 degrees from the laser axis using $\mathrm{Li}_{2} \mathrm{~B}_{4} \mathrm{O}_{7}$ and $\mathrm{CaSO}_{4}$ TLD detectors recording via 5 different absorber filters the most absorbing of which was $3 \mathrm{~cm}$ of $\mathrm{Ta}$. The detectors were absolutely calibrated using a $\mathrm{Co}^{60} 1.33$ and 1.173 MeV gamma source. The X-ray spectrum was inferred iteratively from the TLD data using a Monte Carlo model to compute the TLD signals from the assumed X-ray spectrum. This is a well established unfolding technique and it gave results for the absolute spectral intensity shown in figure 4 . Similar data were recorded with $350 \mathrm{~J}, 5$ ps pulses in the debris shield configuration as also illustrated in figure 4 and these showed as expected reduced spectral brightness at high photon energy.

In such a spectrum a Maxwellian electron energy distribution gives an $\exp (-\mathrm{hv} / \mathrm{kT})$ spectral shape and the slope of a logarithmic plot gives the temperature of the electrons. As has often been seen in laser plasma studies, the slope of the spectrum in figure 4 changes significantly with increasing photon energy to indicate apparently higher temperature at 
higher photon energies. This shows that the electrons do not have a simple Maxwellian energy distribution and that there is a pronounced 'hot tail' in the distribution. ।

The ITS code was used to model these data also, in this case computing the Bremsstrahlung rather than the $\mathrm{K} \alpha$ yield. For the $0.5 \mathrm{ps}$ data it was found that at photon energies $>1 \mathrm{MeV}$ a relativistic Maxwellian of $\langle\mathrm{E}>=6 \mathrm{MeV}(\mathrm{kT}=2.2 \mathrm{MeV})$ gave a reasonable fit to the measured forward directed spectrum if it contained $1.2 \%$ of the laser energy in a 30 degree cone angle. A relativistic Maxwellian of mean energy $1.5 \mathrm{MeV}$ (temperature $600 \mathrm{keV}$ ) with $12 \%$ of the laser energy in a 30 degree cone gave a rough fit to the lower energy part of the X-ray spectrum below $1 \mathrm{MeV}$. Based on our previously discussed data the expected mean energy is $70 \%$ of the RPP at $10^{20} \mathrm{Wcm}^{-2}$, i.e., $2.6 \mathrm{MeV}$. The data seem to show a minor part of the electron energy spectrum with higher mean energy $(6 \mathrm{MeV})$ and the majority of electrons with a lower mean of $1.5 \mathrm{MeV}$. The total energy in the electrons is more difficult to determine as it requires knowledge of the angular distribution of the $\mathrm{X}$ ray emission. Our data also show evidence of forward peaking of the emission as illustrated in figure 5 which plots data from Si diodes filtered to record a spectrum of mean energy $1 \mathrm{MeV}$, but we do not have sufficiently extensive data for an angular integration. These results may simply indicate a wide range in the power spectrum of intensity at the critical density, enhanced by relativistic self focusing in prepulse formed sub critical density plasma or the hot tail may be specifically associated with electron acceleration in relativistically self focused filaments at sub critical density. Further work is needed to distinguish between these two possibilities. 


\section{Electron energy spectra}

Corroboration of the hot tail in the electron energy spectrum was obtained from magnetic spectrometer measurements of electrons escaping from the target. The detector was nuclear

emulsion recording the tracks of individual electrons. Two spectrometers were placed at 30 degrees and 95 degrees from the laser axis producing spectra from $1 \mathrm{~mm}$ thick Au targets shown in figure 6. The recorded electrons had a mean energy of $7 \mathrm{MeV}$ with maximum energies up to $100 \mathrm{MeV}$ being recorded. The integrated energy per steradian in the forward direction was about $1 \%$ of the laser energy, reflecting significant electron energy loss escaping from the potential well together with collisional energy loss in penetrating through the target.

\section{Photo neutrons and nuclear activation}

The highest energy photons in the Bremsstrahlung spectrum induce nuclear processes which give further quantitative evidence of the spectral brightness at photon energy in the region of $20 \mathrm{MeV}$. Nuclei generally exhibit a giant resonance for photo neutron emission and the magnitude of the cross section and its energy threshold vary with atomic number, but in general the resonance has a band width which is narrow enough to give an estimate of the spectral intensity at its peak from the yield of photo neutrons 9 . This yield is most easily deduced by measuring the characteristic gamma emission of the activated nuclei which result from the emission of photo neutrons, though it can also be determined from activation and gamma emission consequent on capture of the photo neutron by a high $\mathrm{Z}$ nucleus or direct detection of the neutron. Data were obtained with massive high $\mathrm{Z}$ targets including for example a $1 \mathrm{~cm}$ wide $1 \mathrm{~cm}$ long solid target with layers of $\mathrm{Au}, \mathrm{Ni}$, In and $\mathrm{Al}$ which was irradiated with a $5 \mathrm{ps} 300 \mathrm{~J}$ pulse at $10^{19} \mathrm{Wcm}^{-2}$. The yield of photo neutrons in $\mathrm{Ni}^{58}$ was inferred to be $910^{5}$ from measurement of 0.5 and $14 \mathrm{MeV}$ gamma decays of the excited $\mathrm{Ni}^{57}$ nucleus produced by photo-neutron emission. A similar result from $300 \mathrm{~J}$ irradiation of an $\mathrm{Au} / \mathrm{Cu}$ target gave $4.510^{7}$ photo-neutrons from $\mathrm{Cu}$. Assuming a slowly 
varying spectral intensity in the vicinity of the giant resonance these data give the spectral intensity at $18.5 \mathrm{MeV}$ and $21.8 \mathrm{MeV}$ respectively which are plotted in figure 4! There is fair agreement with extrapolation of the Bremsstrahlung spectra for both the 5 ps and 0.5 ps cases confirming the hot tail feature in both those spectra.

In summary our observations of hot electron production have shown $28 \%$ lower bound conversion to forward directed electrons of $640 \mathrm{keV}$ mean energy close to the $1 \mathrm{MeV}$ envisaged for fast ignition using intensity $210^{19} \mathrm{Wcm}^{-2}$ and also higher electron energies at higher intensities. This is encouraging particularly when there is evidence of substantial forward peaking of the angular distribution.

\section{Heating}

The second main goal of our research is to measure heating at depth due to the hot electrons. The fast ignitor scheme assumes channel formation at subcritical density and hole boring at super- critical density giving penetration of the laser light to within about two focal spot diameters from the dense fuel. Over a range of this order it is necessary to have minimal lateral spreading of the electron flow and to produce intense heating. Accordingly we looked initially for thermal $x$-ray and thermonuclear neutrons from indicator layers buried at depths up to 50 micron in cold layered solid targets with minimum preformed plasma.

Our modelling base for this work was the 1D Lasnex model discussed previously which predicted heating solid matter at depth up to 20 micron to temperature of the order of $1 \mathrm{keV}$ using 20 ps pulses to generate electrons of shorter range and therefore greater local heating effect. 


\section{$\mathrm{X}$-ray spectroscopy}

As an initial test we recorded streak time resolved $\mathrm{X}$-ray spectra from 1 micront thick layers of $\mathrm{Al}$ with a $\mathrm{CH}$ over-layer of variable thickness using a flat $\mathrm{KAP}$ crystal spectrometer . ${ }^{10}$ The targets were irradiated with $20 \mathrm{ps} 20 \mathrm{~J}$ pulses focused to a 15 micron focal spot. The results showed resonance spectral lines of hydrogenic and $\mathrm{He}$-like $\mathrm{Al}$ with strong spectral broadening. Fitting computed spectra suggested density of 0.8 to $1.210^{23}$ electrons/cc and temperature up to $300 \mathrm{eV}$ to $600 \mathrm{eV}$ for 5 micron over-layer thickness. 2D Lasnex modelling shows that ablative modification of the over-layers was not negligible in this work, where the ASE prepulse was estimated at $25 \mathrm{~mJ}$ and a similar energy was present in a leakage pulse $4 \mathrm{~ns}$ before the main pulse. The fact that the density deduced from the line broadening with the 5 micron over-layer was less than solid density is probably attributable to prepulse effects.

\section{DD nuclear fusion}

An alternative potentially more sensitive measure of the heating of solid matter to $>1 \mathrm{keV}$ temperature is $\mathrm{DD}$ fusion in solid $\mathrm{CD}_{2}$. Experiments were therefore conducted with targets incorporating $\mathrm{CD}_{2}$ layers. Scintillator photo-multiplier neutron detectors were set up at a variety of angles and distances. Maximum sensitivity and high time of flight resolution was afforded by a specialized detector Lansa comprised of a 960 element array of photomultiplier/scintillator detectors, the whole array being screened by $15 \mathrm{~cm}$ of lead shot. The Lansa array was located at $21 \mathrm{~m}$ from the target an angle of 260 degrees to the laser axis. Its signals were converted electronically to a digital form giving the time above a pre defined threshold signal on each channel. The other neutron detectors were single scintillator photo-multiplier units with analog recording on fast oscilloscopes.

Targets were irradiated with $20 \mathrm{ps}$ and $5 \mathrm{ps}$ pulses, the lower electron energy being predicted to maximize the heating close to the focal spot. The experimental data revealed 
features attributable to the hot tail of the electron velocity distribution. Firstly strong signals were detected by Lansa from thin (100 micron) $\mathrm{CH}$ targets with no $\mathrm{CD}_{2}$. The neutron energy spectrum deduced from the timing of the pulses at the detectors was broad, from as high as $10 \mathrm{MeV}$ with the signal level rising continuously to energies below $1 \mathrm{MeV}$, the limit of the range of measurement. This continuum in the time of flight spectra we attribute to $\mathrm{m}$ photo neutron production in the walls of the target chamber. Adding a 10 micron thick layer of $\mathrm{CD}_{2}$ on the surface of the target and placing a 100 micron thick disc of solid $\mathrm{CD}_{2}$ normal to the surface to intercept 1 sterad of the ion emission from the target, gave a measure of $\mathrm{DD}$ fusion due to three causes - fast ions striking the $\mathrm{CD}_{2}$ block, fast ions driven into the target by light pressure and thermal fusion due to heating by electrons. The DD fusion signature was a relatively weak feature on top of the continuum in the time of flight spectrum. The DD yield was $1.710^{6}$ and was seen to be about $10 \mathrm{x}$ broader than the instrumental width for circa $1 \mathrm{keV}$ thermal fusion. It was approximately centered at $\mathbf{2 . 7}$ $\mathrm{MeV}$ suggesting that the dominant beam target interaction was from ions directed away from the target.

It was not possible to unambiguously detect thermal fusion but a variety of targets incorporating $\mathrm{CD}_{2}$ layers were irradiated with both 20 ps and 5 ps pulses and neutron emission in broad energy bands was seen with both Lansa and with the simpler scintillator detectors. An upper bound figure for thermal fusion events from the Lansa data from a 10 micron $\mathrm{CD}_{2}$ layer buried below 0.5 micron of $\mathrm{Cu}$ and irradiated with $400 \mathrm{~J}$ in 20 ps was determined to be $310^{4}$ from the data shown in figure 7. Lasnex simulations of a 10 micron $\mathrm{CD}_{2}$ layer heated to variable initial temperature during the laser pulse with cooling by conduction and hydrodynamic expansion, gave a scaling of neutron yield with temperature from which the upper bound on the $\mathrm{CD}_{2}$ temperature in the experiments was estimated at $800 \mathrm{eV}$. 
The expected heating from the 1D Lasnex calculations was greater, temperatures over 1 $\mathrm{keV}$ being predicted which should have shown a thermal DD fusion yield of more than $10^{5}$. The apparent failure to heat as efficiently as predicted may attributable to limitations of the Lasnex ID model but more likely is traceable to the high prepulse level in these experiments which created a considerable plasma atmosphere through which the heating beam had to penetrate in order to reach the target. Self focusing and beam spraying in the pre-plasma could significantly increase the area of electron energy deposition in the buried layer of $\mathrm{CD}_{2}$ and reduce the heating. Similarly failure to penetrate significantly beyond the critical density in the preformed plasma could put the source of hot electrons at a significant distance from the $\mathrm{CD}_{2}$ layer.

\section{Preformed plasma}

The capacity of an intense beam to penetrate a pre plasma was studied by irradiating thin $\mathrm{CH}$ targets with $20 \mathrm{~J}$ pulse of both $0.5 \mathrm{ps}$ and $20 \mathrm{ps}$ duration. Optical probing interferometry across the target surface at the second harmonic with a short pulse derived by beam splitting from the main pulse, showed details of the pre-plasma formation as indicated in figure 8 . These experiments demonstrated that $20 \mathrm{~J}, 0.5 \mathrm{ps}$ pulses did not penetrate through foils of initial thickness above 1.7 micron. while $20 \mathrm{~J}, 20 \mathrm{ps}$ pulses penetrated up to 2 thick micron foils. There was clearly prepulse modification of the foils but the data suggest that $>10^{-4} \mathrm{gcm}^{-2}$ of preformed plasma at supercritical density presents a significant barrier capable of preventing hole boring by a short duration $20 \mathrm{~J}$ pulse, underlining the need to avoid significant pre-plasma formation in order to optimize heating. 


\section{Conclusions}

Initial studies with the Nova facility have shown efficient generation of electrons in forward collimated flow and of circa $1 \mathrm{MeV}$ energy of interest for fast ignition. Study of heating by the electrons has suggested a need to minimize pre-plasma production in order to optimize heating which so far has been limited to less than $1 \mathrm{keV}$ and is below expectations from ID modelling.

\section{Acknowledgments}

We are grateful for the support of the Nova operations and engineering teams. Work performed under the auspices of the US Department of Energy by the Lawrence Livermore National Laboratory under Contract No. W-7405-ENG-48.

\section{References}

\footnotetext{
' M Tabak J Hammer M E Glinsky W L Kruer S C Wilks J Woodworth E M Campbell M D Perry Phys Plasmas, 1, 1626, (1994)

${ }^{2}$ Lindl, J. D., Laser Interaction and Related Plasma Phenomena, AIP Conf. Proc. 318 , p. 635, (1993).

3 A Pukhov, J Meyer ter vehn Phys Rev. Lett. 79, 2686 ( 1997)

$M$. D. Perry, Science \& Technology Review page 4, December 1996.

S K B Wharton C Brown B A Hammel S Hatchett M Key J Koch J Moody A Offenberger M Perry M Tabak S Wilks V Yanovsky Bul Am Phys Soc 421969 (1997)

' S C Wilks W L Kruer M Tabak A B Langdon Phys Rev Lett. 691383 (1992)

7 Norreys private communication

A Pukhov, J Meyer ter vehn Phys Rev. Lett. 76, 3975( 1996)

9 B L Berman . Atomic data and Nucl. data tables 15, 319 - 390 (1975).

${ }^{10}$ J. A. Koch, C. A. Back, C. Brown, K. Estabrook, B. A. Hammel, S. P. Hatchett, M. H. Key, J. D. Kilkenny, O. L. Landen, R. W. Lee, J. D. Moody,

A. A. Offenberger, D. Pennington, M. D. Perry, M. Tabak, V. Yanovsky,

R. J. Wallace, K. B. Wharton and S. C. Wilks, Lasers and particle beams (in press)
} 


\section{Figures}

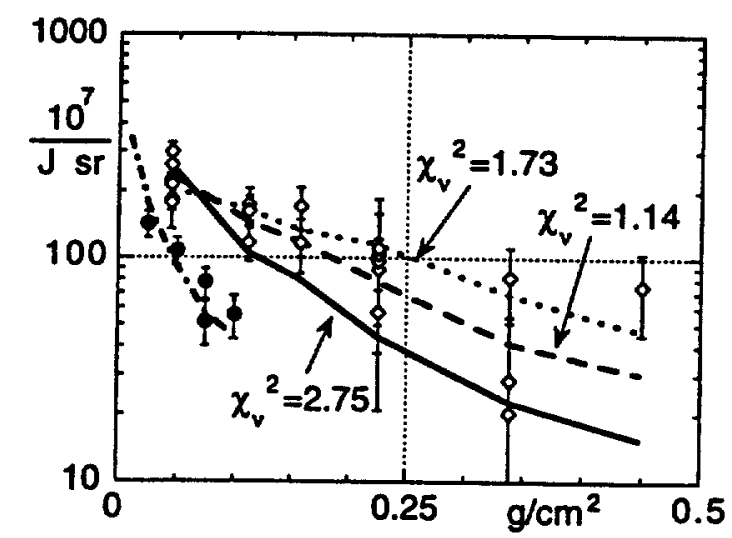

Figure 1. $K_{\alpha}$ signal in units of $10^{7} x$-rays per incident Joule per steradian, plotted against mass per unit area of the front layer of the target. The solid circles are data from $C H$ front-layer targets, empty diamonds are data from $C u$ targets, both at $210^{19} \mathrm{~W} \mathrm{~cm}^{-2}$. The solid line is an ITS fit in $\mathrm{Cu}$ with $E_{o}=330 \mathrm{keV}$ and conversion efficiency $E_{e l e c} / E_{\text {laser }}=308$. The dashed line is $E_{0}=640 \mathrm{keV}$ and $E_{e l e c} / E_{\text {laser }}=298$ The dotted 1 ine is $E_{0}=1040 \mathrm{keV}$ and $E_{\text {elec }} / E_{\text {laser }}=31 \%$. The dash-dot line is an ITS fit in CH for $E_{0}=120 \mathrm{keV}$ and $E_{\text {elec }} / E_{\text {laser }}=298$. 


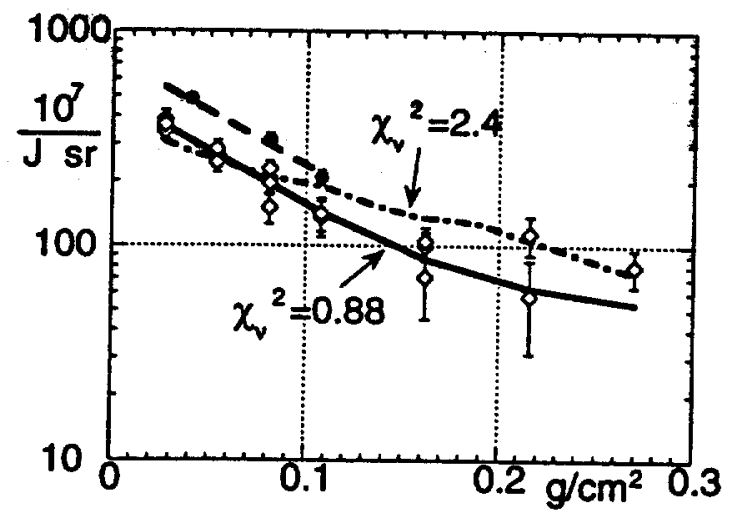

Figure 2. $K_{\alpha}$ signal from the Al targets, in units of $10^{7} \mathrm{x}$ rays per incident Joule per steradian, plotted against mass per unit area of the Aluminum front layer of the target. The solid circles are experimental data at intensities of $410^{19} \mathrm{~W}$ $\mathrm{cm}^{-2}$, empty diamonds are data at $210^{19} \mathrm{~W} \mathrm{~cm}^{-2}$. The solid line is an ITS fit with $E_{0}=330 \mathrm{keV}$ and conversion efficiency $E_{e l e c} / E_{\text {laser }}=318$. The dash-dot line is $E_{0}=640 \mathrm{keV}$ and $E_{\text {elec }} / E_{\text {laser }}=308$ The dashed line is $E_{0}=330 \mathrm{keV}$ and $E_{\text {elec }} / E_{\text {laser }}=478$. 
1

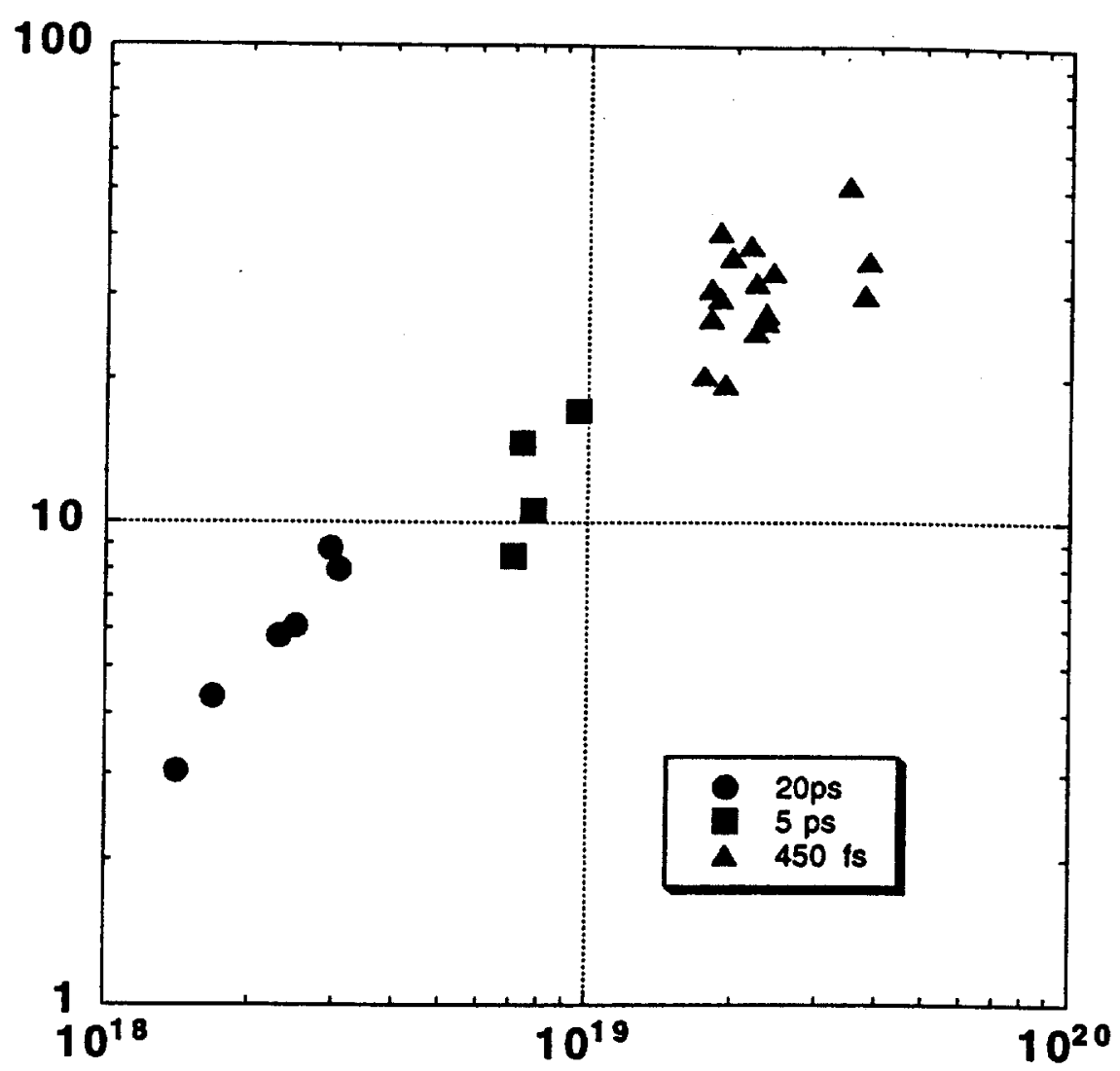

Figure 3. Ordinate efficiency $E_{\text {lectron }} / E_{\text {laser }}$, abscissa intensity on target $W \mathrm{~cm}^{-2} 20$ Ps and 5 ps data are for high energy, 200 to $400 \mathrm{~J}$ and $450 \mathrm{fs}$ data are for 15 to $20 \mathrm{~J}$ 


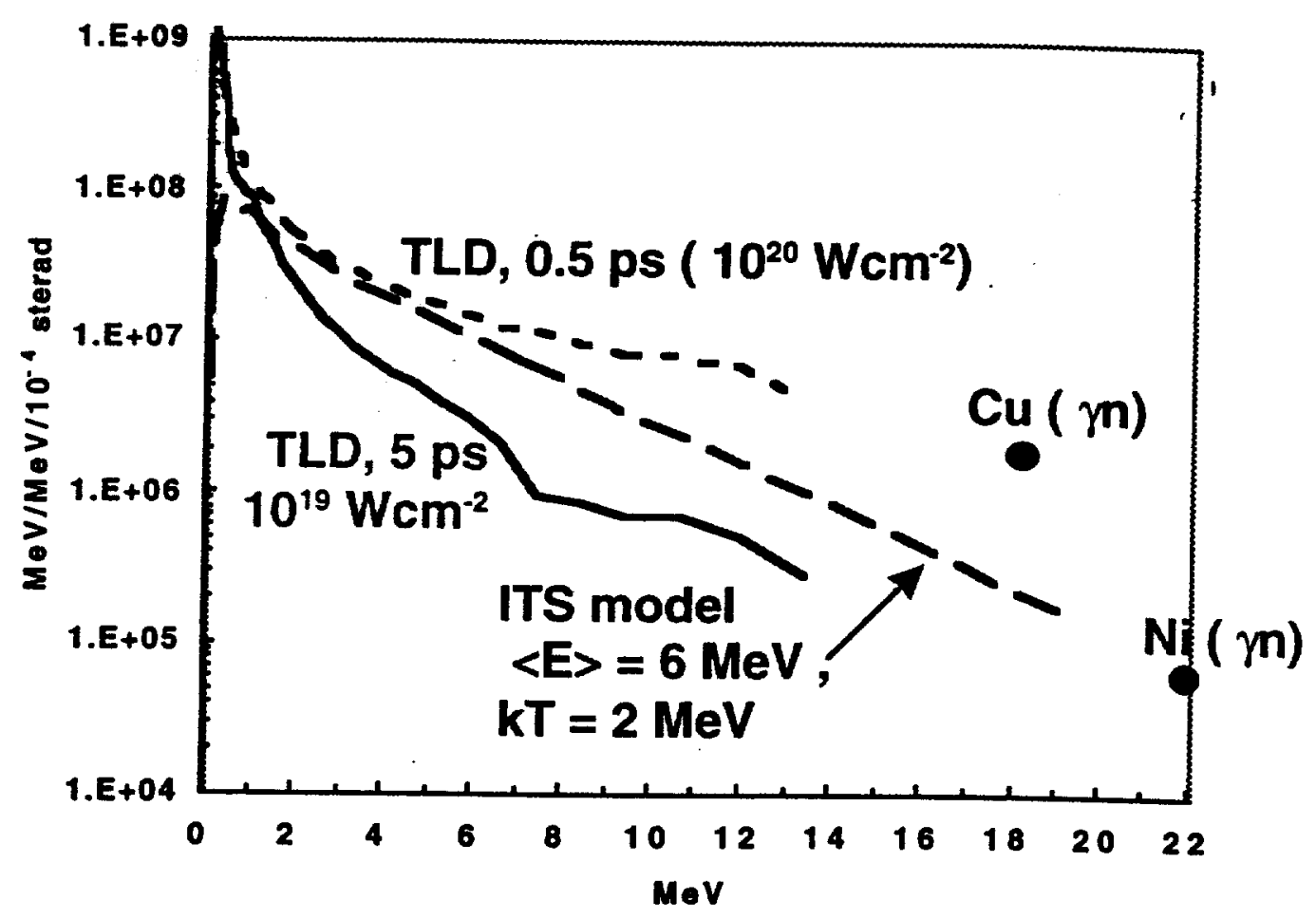

Figure 4 Ordinate; $X$-ray intensity MeV/MeV in 10-4 sterad. Abscissa ; photon energy Spectral data from TLD measurements as labeled. ITS model data as labeled and activation data as labeled 
90

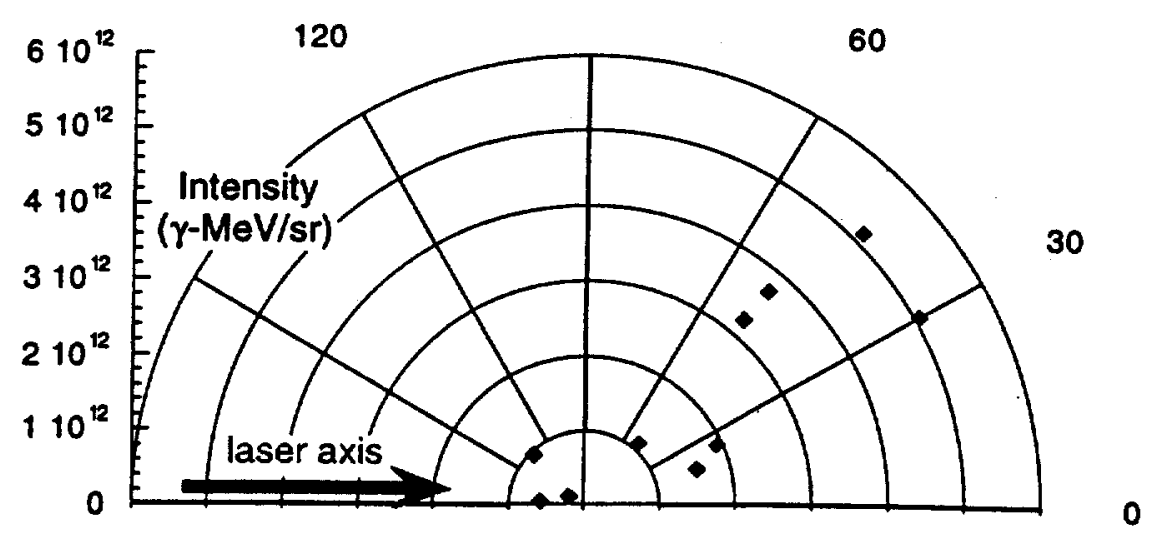

Figure 5 Polar plot of $X$-ray intensity in MeV/sterad for $0.5 \mathrm{ps}$, squares, and 5 ps pulse length diamonds for $300 \mathrm{~J}$ pulse incident on thick Au targets 


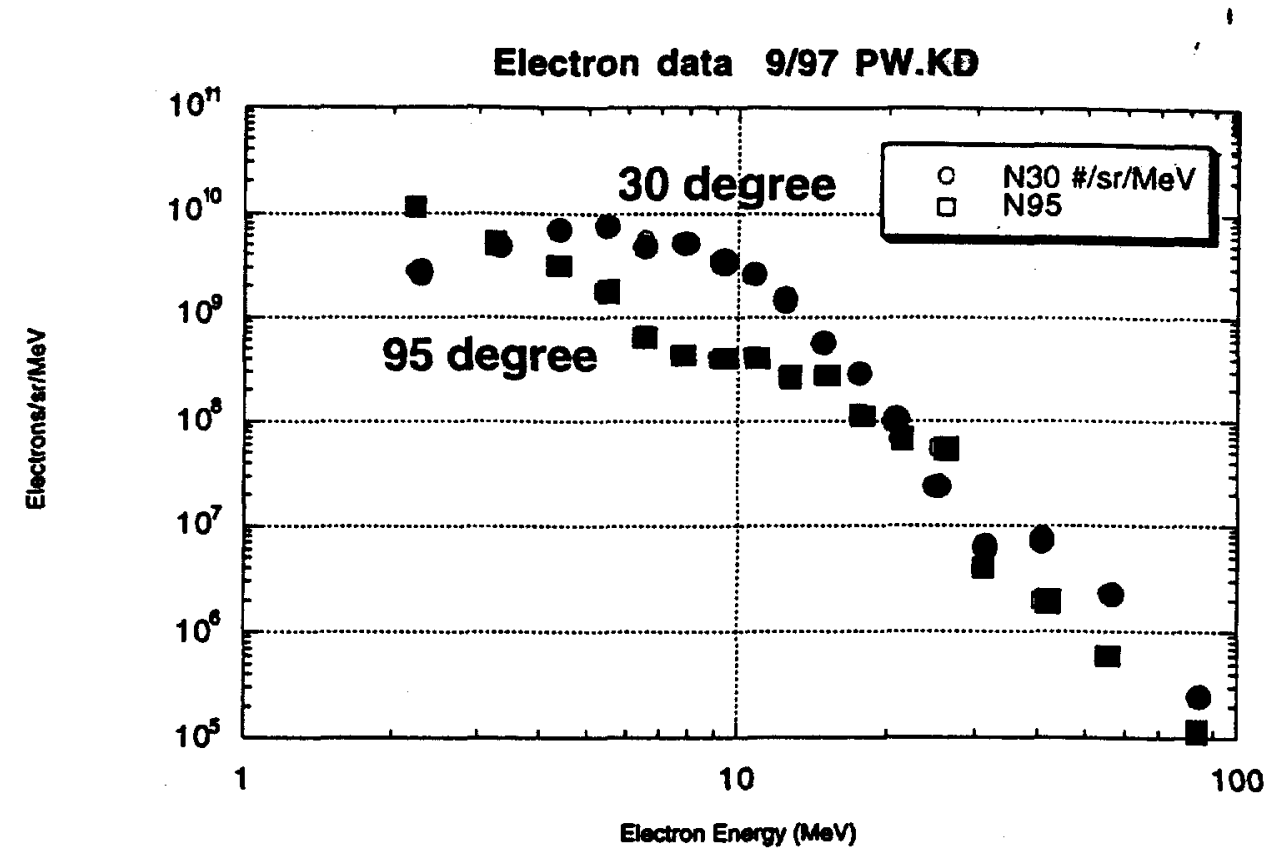

Figure 6. Ordinate; electrons/steradMeV . Abscissa ; electron energy MeV. Data at 30 degrees, filled circles and 90 degrees, filled squares, to the laser axis 


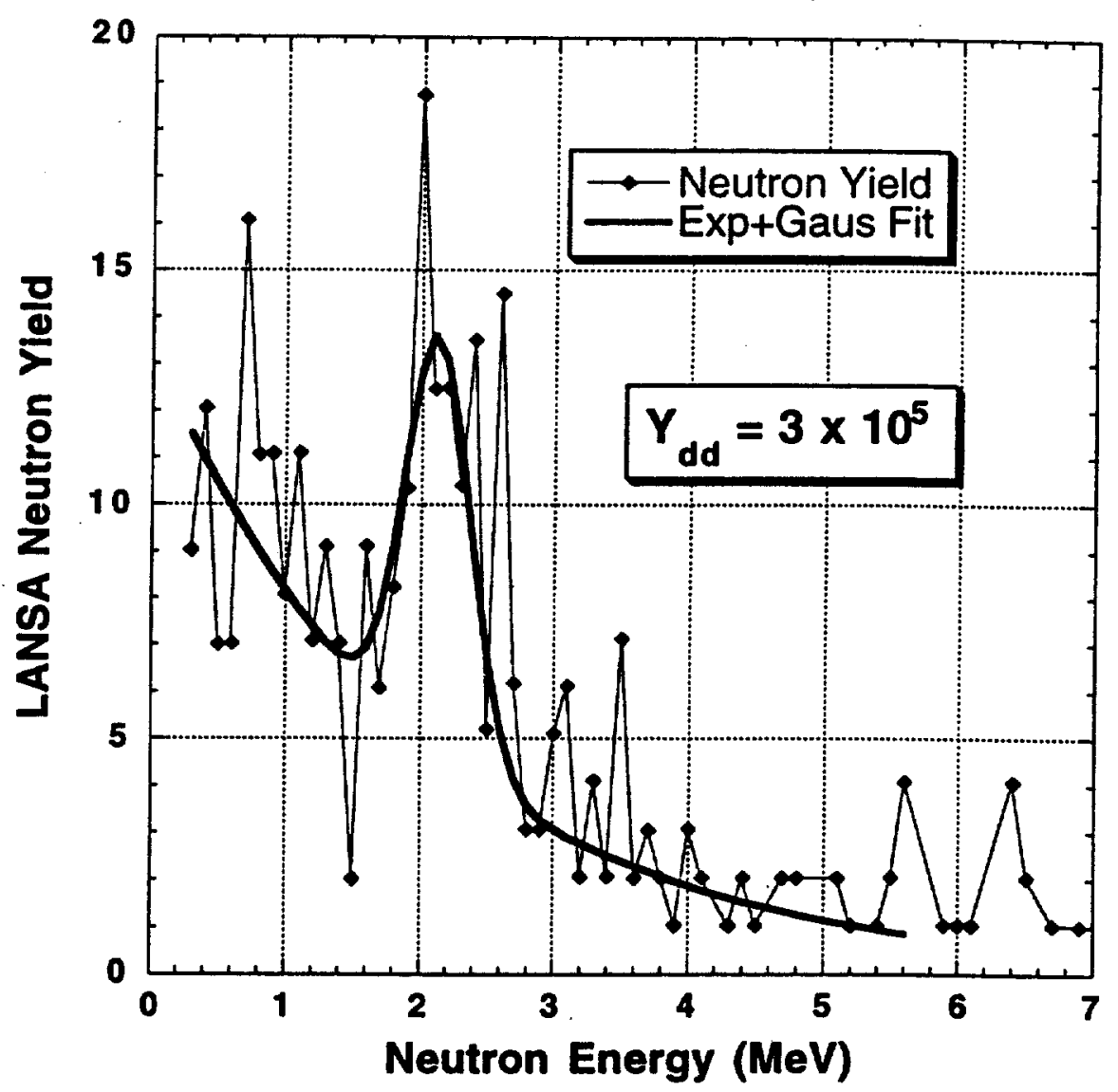

Figure 7 Ordinate; neutron yield in arbitrary units . Abscissa ; neutron energy . The DD fusion feature is seen above a continuum due to photo - neutron emission at the target chamber walls 


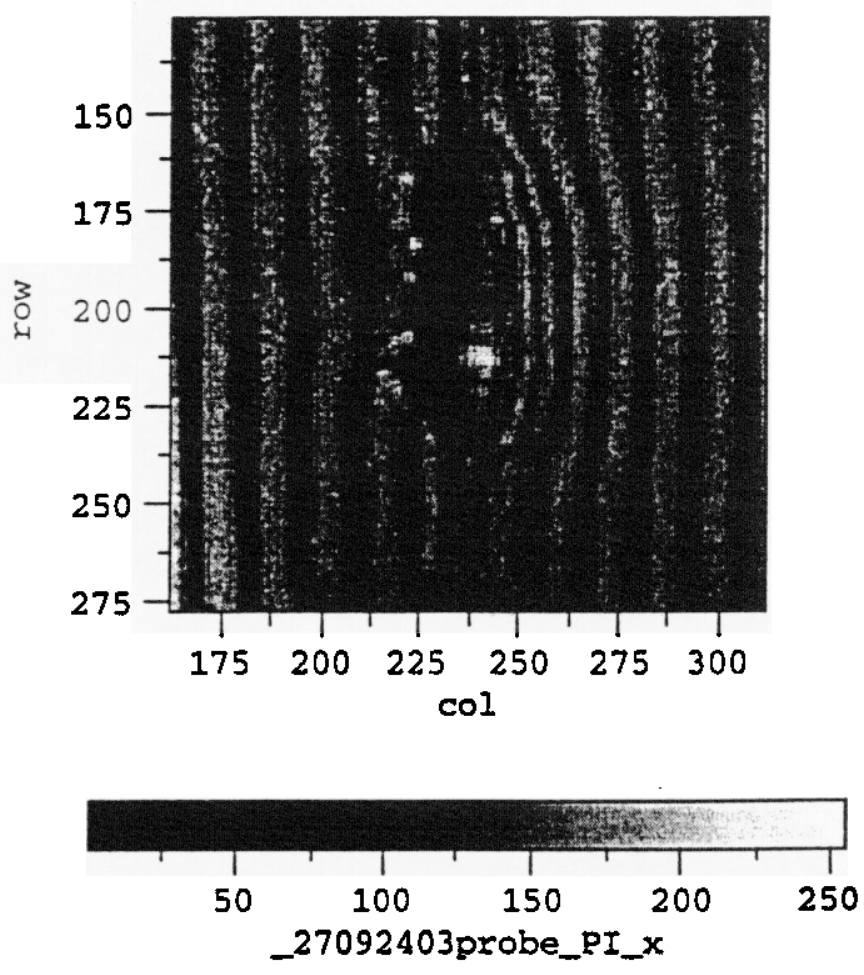

\section{0 micron}

Figure 8 Interferogram recorded with a sub-picosecond pulse of second harmonic light 1ps before the main pulse on a 2 micron $\mathrm{CH}$ target showing plasma formation due to ASE and leakage pulses . 


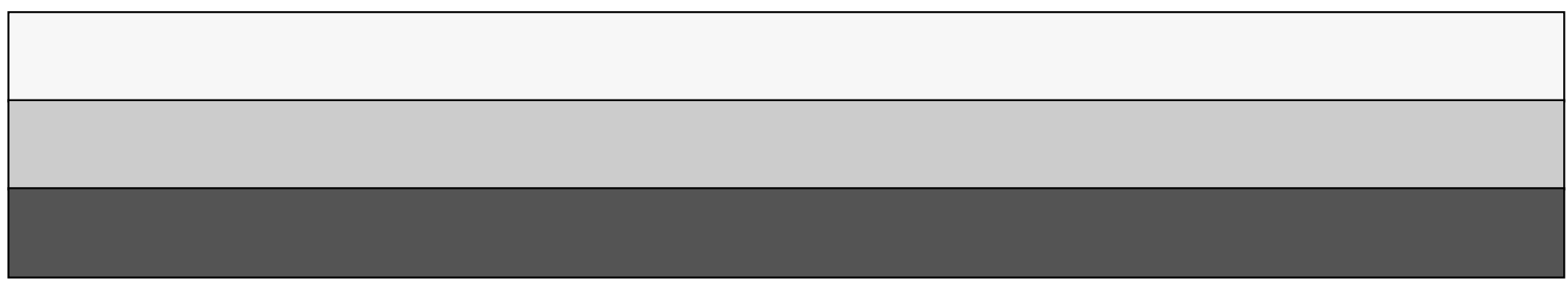

